\title{
PREDICTOR-BASED SELF TUNING CONTROL OF POWER PLANT
}

\author{
Vytautas Kaminskas, Danguolė Janickienė \\ Department of Applied Informatics \\ Vytautas Magnus University, Vileikos 8, LT-44404 Kaunas, Lithuania
}

\begin{abstract}
Design problems of predictor-based self tuning digital control systems for power plants are discussed. Investigation results for self-tuning control algorithms for a nuclear reactor are presented. These algorithms provide the minimum variance of power deviations from the given trajectory in transition and stationary regimes of operation.
\end{abstract}

Keywords: predictor-based self tuning control, generalized minimum variance control, constraints for the control signal, nuclear reactor.

\section{Introduction}

Usually control system design must take into account the fact that a priori information about the plant and its environment is insufficient. Control system must be capable of ensuring the control task despite variations in plant's dynamic and static characteristics, provoked by inner and outer disturbances.

A self-tuning control system usually consists of two loops. The control plant and the controller form the so-called main loop. The second loop may be called the tuning loop, and its aim is to change the control law so as to get adjusted to the unknown situation and to accomplish the control task. Different synthesis methods for the latter loop make it possible to group self-tuning control systems into explicit and implicit ones. An explicit self-tuning control system is based on the estimation of an explicit control plant model, while an implicit one is based on implicit estimation of the controller parameters. Different modifications of self-tuning control systems can be successfully used in order to cope with these requirements $[1,5,6]$.

This paper considers practical issues in the implementation of predictor-based self-tuning control systems $[3,4,8,11]$. In this case, control plant model is constructed in the form of an optimal predictor of the output signal

$$
y_{t+\tau+1}=y_{t+\tau+1 \mid t}(c)+\xi_{t+\tau+1},
$$

where $y_{t+\tau+1 \mid t}(c)$ is optimal $(\tau+1)$ step prediction of the output signal $y_{t+\tau+1} ; c$ is the vector of control plant model parameters; $\xi_{t+\tau+1}$ is a sequence of random values.
In case of a stochastic control plant it is natural to demand for the control system to provide the minimum variance of the deviations of the observed output sequence $y_{t}$ from the reference sequence $y_{t}^{*}$.

Sometimes it is preferable to apply a generalized minimum variance control algorithm, obtained by introducing control costing. In this case, the control criterion is

$$
\begin{array}{r}
Q\left(u_{t+1}\right)=M\left\{\left(y_{t+\tau+1}-y_{t+\tau+1}^{*}\right)^{2}+\right. \\
\left.+\left[L\left(z^{-1}\right) u_{t+1}\right]^{2}\right\},
\end{array}
$$

and optimal control values can be obtained by

$$
u_{t+1}^{*}: Q\left(u_{t+1}\right) \rightarrow \min _{u_{t+1} \in \Omega_{u}},
$$

where

$$
\begin{array}{r}
\Omega_{u}=\left\{u_{t+1}: u_{\text {min }} \leq u_{t+1} \leq u_{\max },\right. \\
\left.\left|u_{t+1}-u_{t}^{*}\right|<\delta_{t}\right\}
\end{array}
$$

is the admissible domain for the control values; $u_{\min }$, $u_{\max }$ are control signal boundaries; $\delta_{t}>0$ are the restriction values for the introduction rate of the control signal; $y_{t}^{*}$ denotes the reference trajectory for the output signal; $L\left(z^{-1}\right)$ is the polynomial weight coefficient; $M$ is the mathematical expectation sign.

Solution of the extremal problem (3) requires the knowledge of genuine plant model parameters $c$. Since these parameters are usually a priori unknown and vary in the operation process, current estimates can be used instead of genuine parameters. The estimates can be obtained in the identification process from the condition [7] 


$$
\hat{c}_{t}: Q^{*}(c)=\frac{1}{t} \sum_{l=1}^{t} \varepsilon_{l \mid l-1}^{2}(c) \rightarrow \min _{c \in \Omega_{c}},
$$

where $\Omega_{c}$ is the admissible domain for the parameters $c$, usually the same as the stability domain for the closed-loop system;

$$
\varepsilon_{t+1 \mid t}(c)=y_{t+1}-y_{t+1 \mid t}(c)
$$

is the error of one step prediction of the output signal; $y_{t+1 \mid t}(c)$ is the optimal one-step-prediction of the output signal.

Thus we arrive at an explicit predictor-based selftuning control system. Its structural diagram is given in Figure $1[8,9]$.

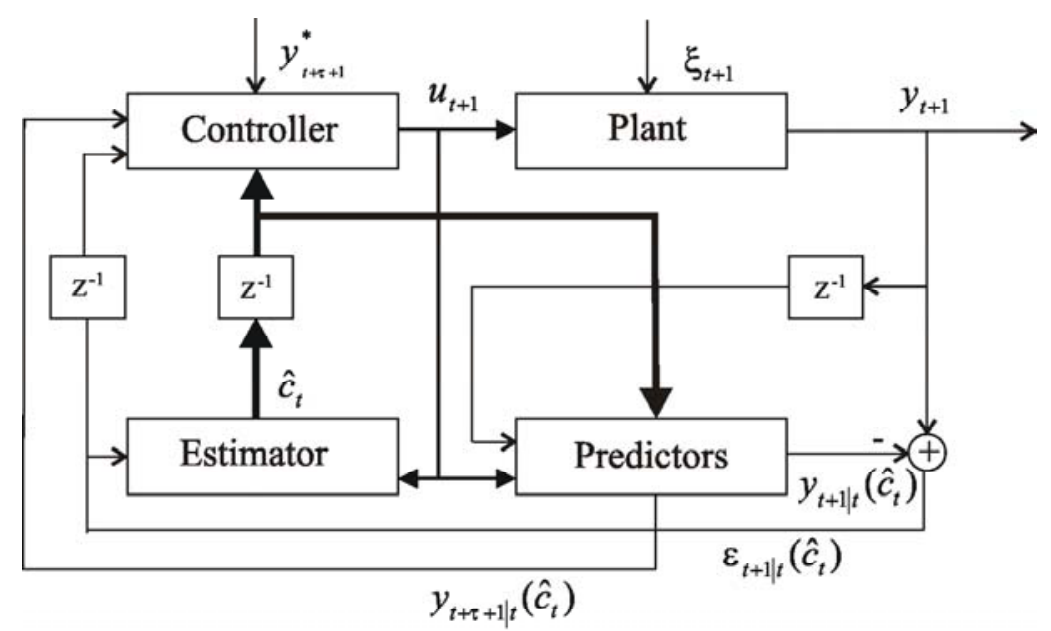

Figure 1. Structural diagram of predictor-based self-tuning control system

\section{Problem statement}

Power plant is a nuclear reactor working in ultimately low power levels and operating as a part of a device assembly designed for physical modelling of the activate zone of a power nuclear reactor. Distinct features of a research-purpose reactor as a control plant are, first of all, possibly frequent variations of the active zone geometry and physical fuel composition, and, secondly, pronounced stochastical character of the chain-reaction in ultimately low power levels distinquishable in the form of strong neutron flux fluctations.

For these reasons, design of control systems, capable of compensating random power fluctuations and of adapting themselves to the variations of the characteristics of the research-purpose reactor, is important.

In the terms of power control system design operational conditions of a research-purpose reactor can be defined by a different stochastic equation

$$
\begin{aligned}
& A\left(z^{-1}\right) P_{t}=z^{-1} B\left(z^{-1}\right) \rho_{t}+\zeta_{t}, \\
& A\left(z^{-1}\right)=1+\sum_{i=1}^{n_{a}} a_{i} z^{-1}, B\left(z^{-1}\right)=\sum_{i=0}^{n_{b}} b_{i} z^{-i},
\end{aligned}
$$

where $\rho_{t}$ and $P_{t}$ are correspondingly introduced reactivity and power values at discrete time moments, $\zeta_{t}$ is the sequence of independent random values with zero mean and finite variance $\sigma_{\zeta}^{2}, z^{-1}$ is onestep delay operation in time domain.
Optimal control law - current control signal values $\rho_{t}^{*}$ is defined from the condition

$$
\begin{gathered}
\rho_{t}^{*}: Q\left(\rho_{t}\right)=M\left\{\left(P_{t+1}-P_{t+1}^{*}\right)^{2}+\right. \\
\left.+\left[L\left(z^{-1}\right) \rho_{t}\right]^{2}\right\} \rightarrow \min _{\rho_{t} \in \Omega_{\rho}}, \\
\Omega_{\rho}=\left\{\rho_{t}: \rho_{\min } \leq \rho_{t} \leq \rho_{\max },\right. \\
\left.\left|\rho_{t}-\rho_{t-1}^{*}\right| \leq \delta_{\rho}\right\},
\end{gathered}
$$

where $P_{t}^{*}$ is reference power value at the moment $t$; $\rho_{\min }, \rho_{\max }$ are possible reactivity value limits, $\delta_{\rho}$ is the restriction value for the reactivity introduction rate, defined in accordance with nuclear safety rules.

\section{Control algorithms}

Optimal reactivity, satisfying the law (9), is defined from the relationship [8]

$$
\rho_{t}^{*}=\left\{\begin{array}{lll}
\min \left\{\rho_{\max }, \rho_{t-1}^{*}+\delta_{t}, \tilde{\rho}_{t}\right\} & \text { if } & \widetilde{\rho}_{t} \geq \rho_{t-1}^{*}, \\
\max \left\{\rho_{\min }, \rho_{t-1}^{*}-\delta_{t}, \widetilde{\rho}_{t}\right\} & \text { if } & \widetilde{\rho}_{t}<\rho_{t-1}^{*}
\end{array}\right.
$$

Reactivity values $\widetilde{\rho}_{t}$ in control law (11) are defined with the methods described below.

Control criterion $Q\left(\rho_{t}\right)$ can be rewritten as

$$
\begin{aligned}
Q\left(\rho_{t}\right) & =M\left\{\left(P_{t+1 \mid t}-P_{t+1}\right)^{2}+\right. \\
& \left.+\left[L\left(z^{-1}\right) \rho_{t}\right]^{2}\right\}+\sigma_{\zeta}^{2},
\end{aligned}
$$


where the optimal one-step-ahead power prediction can be defined by a recursive equation [1]

$$
P_{t+1 \mid t}=F\left(z^{-1}\right) P_{t}+B\left(z^{-1}\right) \rho_{t},
$$

where polynomial $F\left(z^{-1}\right)$ is defined from the relationship

$$
\begin{aligned}
& 1=A\left(z^{-1}\right)+z^{-1} F\left(z^{-1}\right), F\left(z^{-1}\right)=\sum_{i=0}^{n_{a}} f_{i} z_{i}, \\
& \sigma_{\zeta}^{2}=M\left\{\left(\zeta_{t+1}\right)^{2}\right\}, \zeta_{t+1}=P_{t+1}-P_{t+1 \mid t} .
\end{aligned}
$$

In order to simplify calculations in defining optimal control signal values, a generalized output function $\phi_{t}$ is introduced [2]. Its optimal one-stepahead prediction can be defined by the following equation, minimizing the criterion (12) in the following way

$$
\begin{gathered}
\phi_{t+1 \mid t}=\frac{\partial Q\left(\rho_{t}\right)}{\partial \rho_{t}}=P_{t+1 \mid t}+ \\
+D\left(z^{-1}\right) \rho_{t}-P_{t+1}^{*}=0
\end{gathered},
$$

where

$$
D\left(z^{-1}\right)=l_{0} L\left(z^{-1}\right) / b_{0}
$$

and correspondingly,

$$
\phi_{t+1}=P_{t+1}+D\left(z^{-1}\right) \rho_{t}-P_{t+1}^{*} .
$$

Optimal control law, obtained by minimizing the control criterion

$$
\begin{array}{r}
Q\left(\rho_{t}\right)=M\left\{\phi_{t+1}^{2}\right\}=M\left\{\left(\phi_{t+1 \mid t}+\zeta_{t+1}\right)^{2}\right\}= \\
=\left(\phi_{t+1 \mid t}\right)^{2}+\sigma_{\zeta}^{2}
\end{array},
$$

coincides with the control law obtained by minimizing the criterion (12).

In minimizing the criterion $Q\left(\rho_{t}\right)$, a prediction can be obtained by the following recursive equation

$$
\phi_{t+1 \mid t}=F\left(z^{-1}\right) P_{t}+G\left(z^{-1}\right) \rho_{t}-P_{t+1}^{*},
$$

where

$$
G\left(z^{-1}\right)=B\left(z^{-1}\right)+D\left(z^{-1}\right)
$$

has zero value every time step and drives the generalized output

$$
\phi_{t+1}=\phi_{t+1 \mid t}^{*}+\zeta_{t+1}=x_{t}^{T} c+\zeta_{t+1}
$$

to a white noise sequence.

In the equation (22),

$$
x_{t}^{T}=\left\{P_{t}, P_{t-1}, \ldots ; \rho_{t}, \rho_{t-1}, \ldots ; P_{t+1}^{*}\right\}
$$

represents the measurement vector and

$$
c^{T}=\left\{f_{0}, f_{1}, \ldots ; g_{0}, g_{1}, \ldots ;-1\right\}
$$

represents the vector of unknown parameters.

Control law obtained in this case

$$
\tilde{\rho}_{t}=G^{-1}\left(z^{-1}\right)\left[F\left(z^{-1}\right) P_{t}-P_{t+1}^{*}\right]
$$

contains parameters depending on the control plant parameters, which are a priori unknown and can vary in the operating process. The unknown genuine parameters $c$ can be substituted by their current estimates $\hat{c}_{t}$ obtained in the identification process (5), where

$$
\varepsilon_{t+1 \mid t}(c)=\phi_{t+1}-x_{t}^{T} c \text {. }
$$

Such a design approach, the so-called implicit selftuning control scheme allows to obtain the controller parameters directly without obtaining the control plant parameters first, and that simplifies the control signal calculations. The drawback of the method is that it can be applied only to minimum-phase systems and to those nonminimum-phase systems that can give the roots of the closed-loop characteristic equation

$$
B\left(z^{-1}\right)+D\left(z^{-1}\right) A\left(z^{-1}\right)=0
$$

outside the unit circle on the z-plane by choosing the appropriate parameters of the polynomical $D\left(z^{-1}\right)$.

For this reason, equation (25) gives good results for research-purpose reactor's operation in stationary and transition regimes for small reactivity values. In the speed-up regime of the research-purpose reactor with significant reactivity values, the process becomes nonstationary, and in the closed-loop system unobservable and unstable regimes may turn up, manifesting themselves on the control signal dynamics.

In this case the system under identification becomes a nonminimum-phase one, and the polynomial of weight coefficients $D\left(z^{-1}\right)$ doesn't ensure the system's stability. Choosing too large polynomial parameter values results in control quality.

In this case, the control task can be solved by applying control scheme with control plant parameter identification, instead of direct adaptive control. Parameters under identification are the parameters of the polynomials $A\left(z^{-1}\right)$ and $B\left(z^{-1}\right)$, and the factorization method is applied in case of an unstable polynomial $B\left(z^{-1}\right)[1]$.

Control criterion (9), when $L\left(z^{-1}\right)=0$, is substituted by

$$
Q\left(\rho_{t}\right)=M\left\{P_{t+1}-P_{t+1}^{*}\right\}^{2}
$$

and the vector of the parameters of the generalized output function under minimization becomes

$$
c^{T}=\left\{-a_{1},-a_{2}, \ldots,-a_{n_{a}}, b_{0}, \ldots, b_{n_{b}},-1\right\} .
$$

Application of the factorization method is accomplished in the following way.

Polynomial $B\left(z^{-1}\right)$ is decomposed into two factors 


$$
B\left(z^{-1}\right)=B_{+}\left(z^{-1}\right) B_{-}\left(z^{-1}\right),
$$

where

$$
B_{+}\left(z^{-1}\right)=\sum_{i=0}^{n_{b}^{+}} b_{i}^{+} z^{-i}
$$

roots of the polynomial (31) are outside the unit circle,

$$
B_{-}\left(z^{-1}\right)=1+\sum_{i=1}^{n_{\bar{b}}^{-}} b_{i}^{-} z^{-i},
$$

roots of the polynomial (32) are inside the unit circle or on its boundary.

In this case, the control law can be rewritten

$$
\begin{array}{r}
\tilde{\rho}_{t}=-E^{-1}\left(z^{-1}\right)\left[B_{+}^{-1}\left(z^{-1}\right) F\left(z^{-1}\right) P_{t}-\right. \\
\left.-B^{-1}\left(z^{-1}\right) \widetilde{B}_{-}\left(z^{-1}\right) P_{t+1}^{*}\right],
\end{array}
$$

where the polynomials $E\left(z^{-1}\right)$ and $F\left(z^{-1}\right)$ satisfy the relationship

$$
\begin{array}{r}
\widetilde{B}_{-}\left(z^{-1}\right)=A\left(z^{-1}\right) E\left(z^{-1}\right)+ \\
+z^{-1} B_{-}\left(z^{-1}\right) F\left(z^{-1}\right)
\end{array}
$$

and

$$
\widetilde{B}_{-}\left(z^{-1}\right)=\sum_{i=0}^{n_{b}^{-}} b_{n-i}^{-} z^{-i}, \quad n=n_{b}^{-} .
$$

\section{Identification algorithms}

In the stabilization and power elevation, regimes of the research-purpose reactor for small control signal values current values $\hat{c}_{t}$, used in the control algorithm instead of unknown parameters $c$, are received in identification process in the closed-loop with help of the recursive least squares method

$$
\begin{aligned}
& \hat{c}_{t+1}=\hat{c}_{t}+k_{t+1} \varepsilon_{t+1 \mid t}\left(\hat{c}_{t}\right), \\
& k_{t+1}=\Pi_{t} x_{t+1}\left(1+x_{t+1}^{T} \Pi_{t} x_{t+1}\right)^{-1}, \\
& \Pi_{t+1}=\left(I-k_{t+1} x_{t+1}^{T}\right) \Pi_{t}, \\
& \Pi_{n_{c}}=\left[\sum_{t=1}^{n_{c}} x_{t} x_{t}^{T}\right]^{-1},
\end{aligned}
$$

where $I$ stands for the unit matrix; $n_{c}$ is the number of unknown parameters.

In the speed-up regime of the research-purpose reactor, for significant control signal values and fast parameter changes, the algorithm (36)-(39) is modified by means of the restricted exponential forgetting method, which allows to suppress the old information and to increase the numerical reliability of identification [10].

Equation (38) is rewritten as

$$
\Pi_{t+1}=\left(I-\lambda_{t+1} x_{t+1}^{T}\right) \Pi_{t},
$$

where

$$
\begin{aligned}
& \lambda_{t+1}=\gamma_{t} \Pi_{t} x_{t+1}\left(1+\gamma_{t} x_{t}^{T} \Pi_{t} x_{t}\right), \\
& \gamma_{t}=\varphi_{t+1}-\left(1-\varphi_{t+1}\right) \zeta_{t}^{-1}, \\
& \zeta_{t}=x_{t}^{T} \Pi x_{t} .
\end{aligned}
$$

The forgetting factor, defined according to the recursive equation

$$
\begin{aligned}
& \varphi_{t+1}^{-1}=1+ \\
& +(1+k)\left[\ln \left(1+\zeta_{t}\right)+\frac{\zeta_{t}}{1+\zeta_{t}}\left(\varepsilon_{n_{t}}^{2}-1\right)\right]
\end{aligned}
$$

depends on the empirically chosen parameter $k$ on the normalized square prediction error of the generalized output

$$
\varepsilon_{n_{t}}^{2}=\frac{\left(v_{t}+1\right) \eta_{t}}{1+\zeta_{t}+\eta}
$$

where

$$
\begin{aligned}
& v_{t+1}=\varphi_{t+1}\left(v_{t}+1\right), \\
& \eta_{t}=\varepsilon_{t}^{T} \mu^{-1} \varepsilon_{t}, \\
& \mu_{t+1}=\varphi_{t+1}\left[\mu_{t}+\frac{\varepsilon_{t} \varepsilon_{t}^{T}}{1+\zeta_{t}}\right] .
\end{aligned}
$$

\section{Simulation results}

Apriori information about the orders of polynomials $n_{a}=n_{b}=2$ and the initial estimate values for the parameters of the research-purpose reactor's mathematical model (7)-(8) were obtained in the process of off-line identification by means of solving the point kinetics equation, taking into account the stochastic character of the neutron fission process.

$$
\begin{aligned}
& A_{0}\left(z^{-1}\right)=1-0,553781 z^{-1}-0,448023 z^{-2}, \\
& B_{0}\left(z^{-1}\right)=0,270381 \cdot 10^{-8}- \\
& -0,145104 \cdot 10^{-8} z^{-1}-0,111483 \cdot 10^{-8} z^{-2}
\end{aligned}
$$

In the stabilization and speed-up for small control values, the control quality criterion was applied in the form

$$
L\left(z^{-1}\right)=\lambda\left(1-z^{-1}\right) .
$$

Figure 2 illustrates process in the nuclear reactor power regime with power increasing up to certain reference level by means of the control algorithm (11), (25) and the algorithm (11), (33) with factorization after 100 second. Unsignifluctuation of the control signal before switching on the factorization is observed.

Figure 3 presents the behaviour of the control and output signals at the speed-up regime of the nuclear reactor corresponding to the control process according to the algorithm with factorization (11), (33) and without it (11), (25) $\lambda=0$ in (51). These figures show 
that fluctuations of the control and output signals are removed after switching on the factorization algorithm

in the twentieth second.

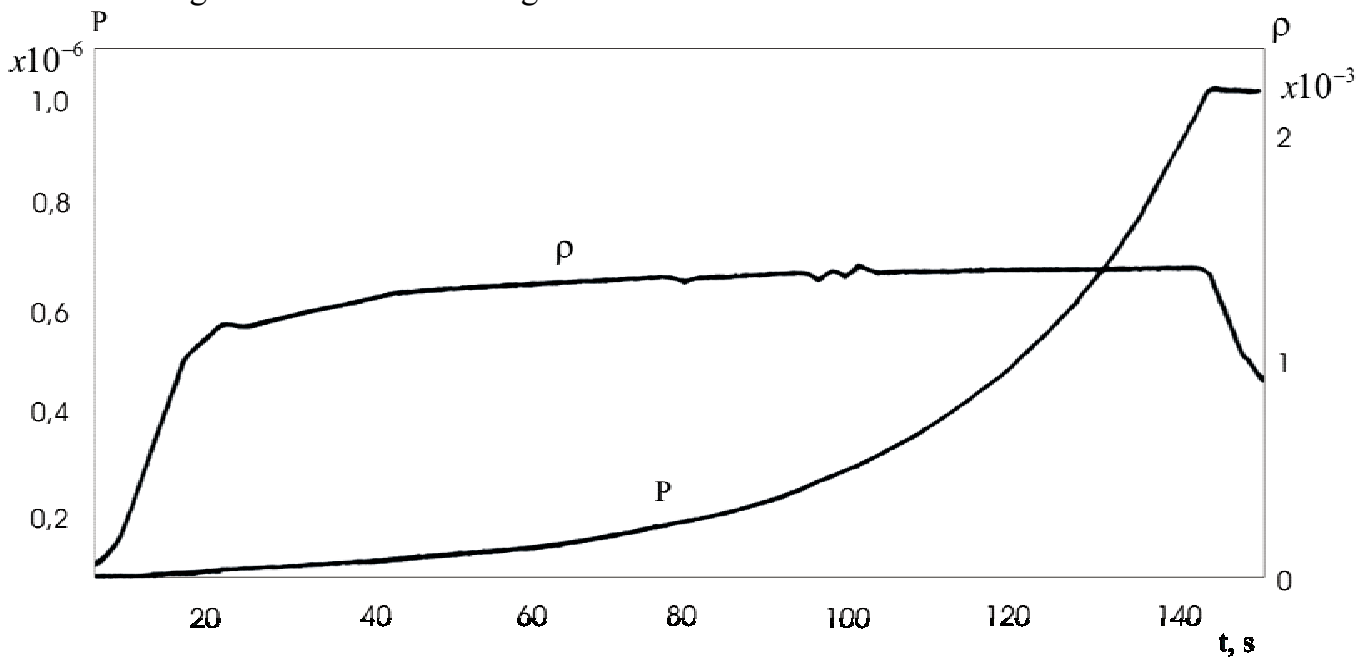

Figure 2. Self-tuning control process for nuclear reactor at the elevation regime

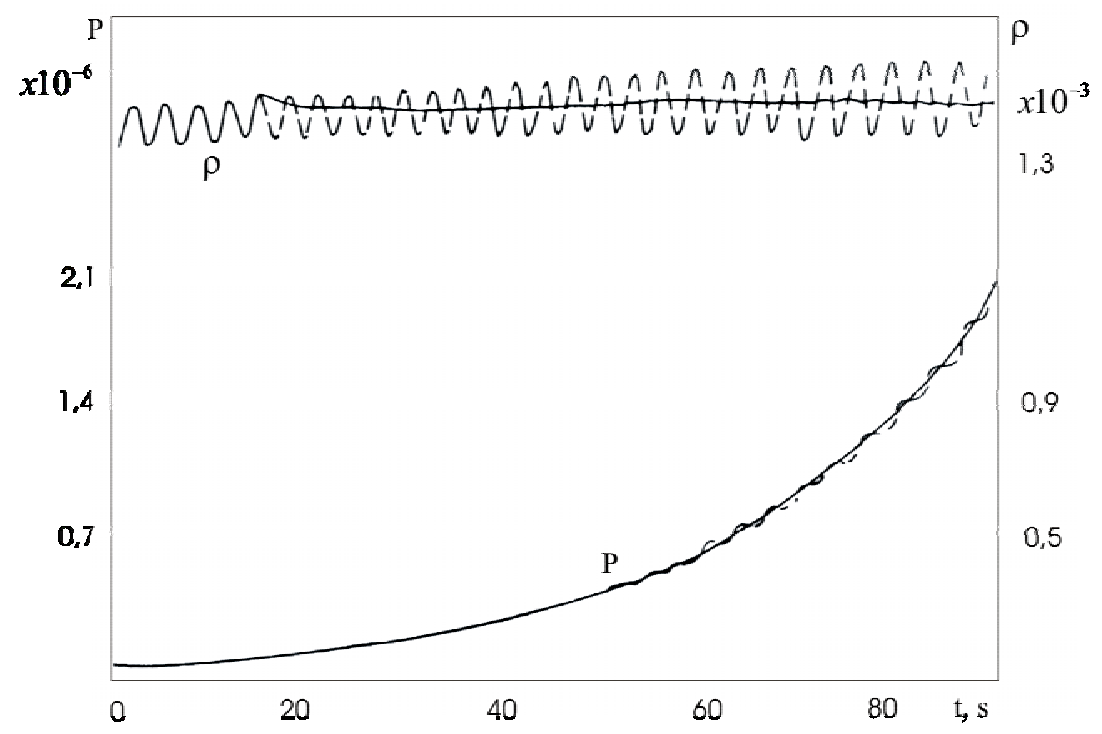

Figure 3. Self-tuning control process for the nuclear reactor at the speed-up regime

\section{Conclusions}

Design problems of predictor-based self-tuning control for power plants are discussed. Control algorithm synthesis is accomplished, taking into account amplitude and/or introduction rate constraints for the control signals. The unknown parameters of the optimal predictor are being estimated in the identification process in the closed loop.

Investigations, that were accomplished, showed the effectiveness of the designed control algorithms for the power control of as nuclear reactor. The algorithms ensure minimal power deviations from the reference trajectory, taking into account amplitude restrictions and variation rate of the control signal in the speed-up and power stabilization regimes. In stabilization and speed-up regimes for small control signal values the direct (implicit) tuning algorithm was applied (immediate adjustment for the parameters of the control law). In the speed-up regime under significant control signal values, the self-tuning control algorithm with identificator and factorization of the unstable polynomial under fast changing parameters was applied, and the factor of exponential forgetting introduced into the identification process is ensured by using the square roots of the covariance matrix.

\section{References}

[1] K.J. Astrom, B. Wittenmark. Computer Controlled Systems - Theory and Design. Prentice Hall, Englewood Cliffs, 1984.

[2] D.V. Clarke, D. Phill, P.J.Gowthrop. Self-tuning controller. Proceeding of the IEE, Vol.122, No.9, 1975, 929-932. 
[3] D.W. Clarke. Self-tuning control of nonminimumphase systems. Automatica, 20(5), 1984, 501-517.

[4] D.W. Clarke, C.Mohtadi, P.S. Tuffs. Generalized predictive control. Automatica, 23(2), 1987, 137-160.

[5] D.W. Clarke. Advances in model predictive control. Oxford, UK, Oxford Science Publication, 1994.

[6] R. Isermann. Digital Control Systems. Springer, Berlin, 1981.

[7] V. Kaminskas. Dynamic System Identification via Discrete-time Observations. Pt. I - Statistical Method Foundations. Estimation in Linear Systems. Mokslas, Vilnius, 1982.
[8] V. Kaminskas. Predictor-based self-tuning control systems. 33 Intern. Wiss. Koll., Heft 1. TH Ilmenau, 1988, 153-156.

[9] V. Kaminskas, D. Janickienė, K. Šidlauskas, D. Vitkutè. Predictor-based self-tuning control systems with constraints. Proceeding of $5^{\text {th }}$ IFAC Symposium an Adaptive Systems in Control and Signal Processing. Budapest, 1995, 101-115.

[10] R. Kulhavy. Restricted exponential forgetting in realtime identification. Automatica, 23(5), 1987, 598-600.

[11] V. Peterka. Predictor-based self-tuning control. Automatica, 19(5), 1984, 471-486.

Received January 2006. 